\title{
Effect of a Coating Induced Residual Stress on Magnetic Domain Structure in Non-Oriented Electrical Steels
}

\author{
Yaoyao Ding ${ }^{1}$, Matthew Gallaugher ${ }^{1}$, Nicolas Brodusch ${ }^{1}$, Raynald Gauvin ${ }^{1}$, and Richard R. Chromik ${ }^{1}$ \\ 1. Department of Mining and Material Engineering, McGill University, Montreal, QC, Canada
}

To produce the magnetic core of the electric motors, non-oriented electrical steels (NOES) are used. Recently much attention has been focused on optimizing the efficiency of electric motors and, as such, various materials aspects of NOES are being re-examined. Typically, NOES are used in the form of laminations ( 200-1000 $\mu \mathrm{m}$ thick) with an electrically insulating coating applied to the surface. Residual stress is induced during the coating process, which will alter magnetic domain structures of the steel substrate. Variations of the resultant magnetic domains due to coating have been characterized mainly in grain oriented electrical steels [1-3], and only limited numbers of studies are available on non-oriented electrical steels [4]. In this study, the effect of the coating is examined, specifically, its role in creating a residual stress near the coating/steel interface. The stress was investigated by nanoindentation technique. With this method, a tensile stress of $200 \mathrm{MPa}$ was calculated based on hardness values in the steel substrate, which was linked to the variations in near surface hardness profile, and corresponding magnetic domain structure. The magnetic domain structures were imaged using a field emission scanning electron microscope (FE-SEM).

From the cross section of a coated sample, crystal orientations were determined by electron backscatter diffraction (Figure 1a) and magnetic domain structures were imaged using the forescatter detector on the electron backscatter diffraction camera, operating in channeling contrast conditions (Figure 1b). A reduction in supplementary domain structure (Figure 1b) was observed within the enclosed zones by the red dashed lines (Figure 1b). Similar features have been reported in previous electrical steel studies [1-4]. With an application of tensile stress, redistribution and increasing in the number of $180^{\circ}$ domain walls were observed along the stress direction. Hence, the reduced complexity of the domain structure indicates the presence of residual stress. The unstressed patterns can be explained by the calculated steel texture parameter $\beta$, where $\beta$ is the angle between the closest magnetic easy axis and the surface of the sample [5]. One nanoindentation hardness profile was produced by indenting (15 x 5 array) within the same region as in Figure 1b. As shown in Figure 1c, hardness obtained from the stressed region was lower than that obtained from the unstressed region within the same grain. This reduced hardness further confirmed the presence of tensile stresses.

The coating stress in NOES was determined by the simplification of the corresponding magnetic domain structures that were directly imaged using FE-SEM, and also validated by the nanoindentation hardness profile. The resultant stressed magnetic domains demonstrated a simpler structure, comparing with these observed from the unstressed region within an individual grain. A greater understanding of residual stress will help to optimize the efficiency of electric motors. 


\section{References:}

[1] N. Chukwuchekwa, A.J. Moses, and P. Anderson, IEEE Transactions on Magnetics 48 (2012), pp. 1393-1396.

[2] B. Fukuda et al, IEEE Transactions on Magnetics MAG-17 (1981), pp. 2878-2880.

[3] S.D. Washko and E.G. Choby, IEEE Transactions on Magnetics MAG-15 (1979), pp. 1586-1591.

[4] K. Senda et al, Electrical Engineering in Japan 182 (2013), pp. 10-18.

[5] N. Bernier et al, Micron 54-55 (2014), pp. 43-51.

a
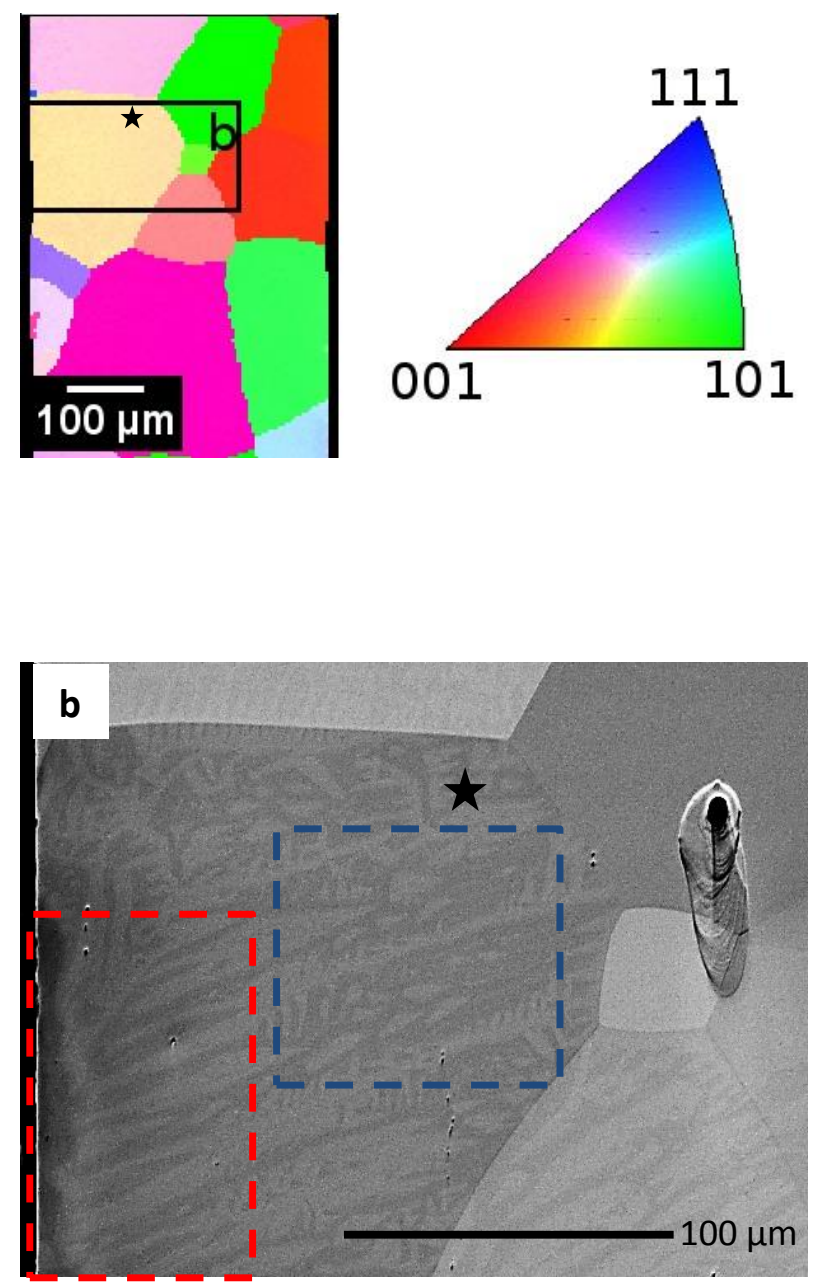
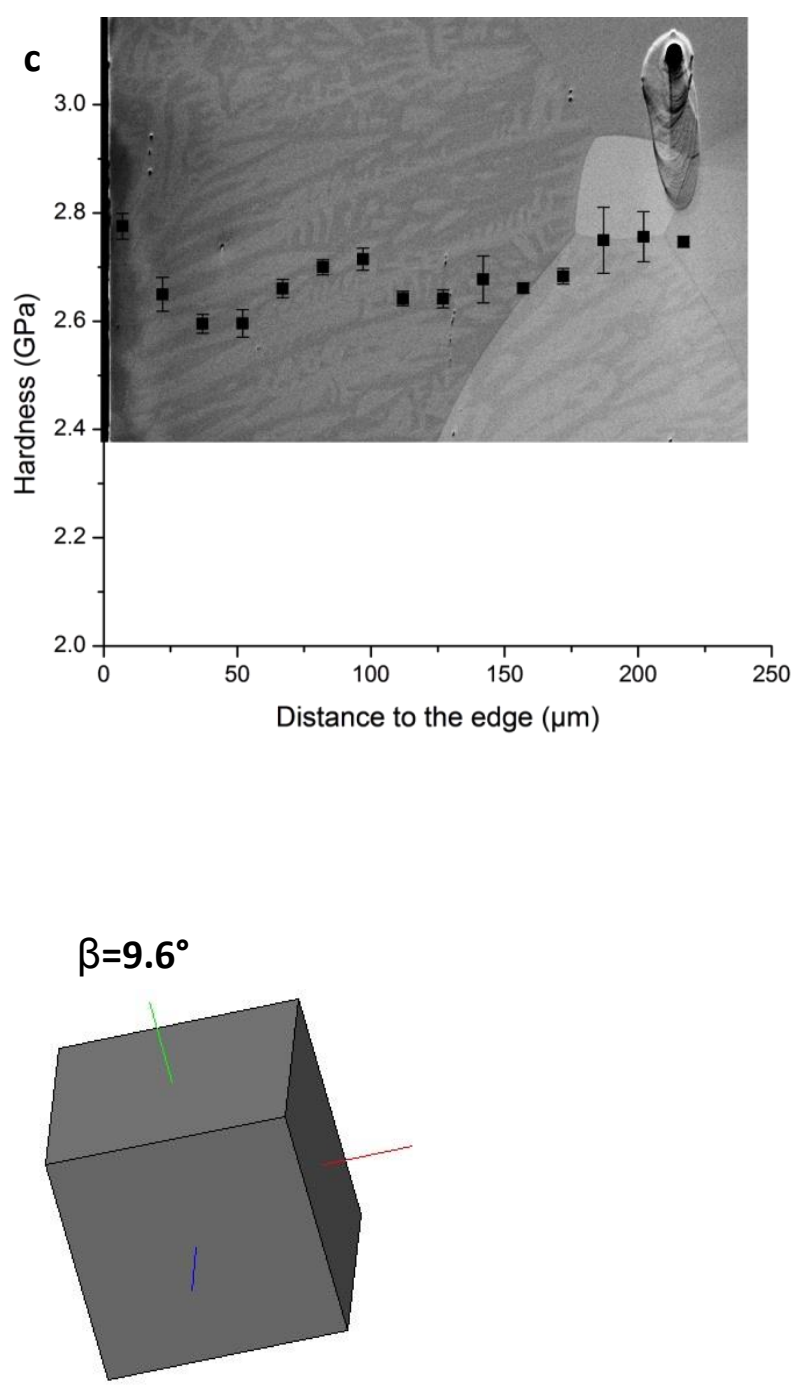

Figure 1. Variation of magnetic domain structures as a result of stress. Crystal orientations were determined by electron backscatter diffraction (a) and illustrated by the crystal on the right (b) for the grain indicated by the star. The steel texture parameter $\beta$ for the same grain was calculated from the orientation [5]. Additionally, the stressed zone determined is outlined by the red dashed box, and the unstressed zone is outlined by the blue dashed box in (b). One nanoindentation hardness profile (c) was obtained from the same area as in (b). 